\title{
The impact of liver fibrosis on microvascular invasion and prognosis of hepatocellular carcinoma with a solitary nodule: a Surveillance, Epidemiology, and End Results (SEER) database analysis
}

\author{
En Lin", Baojia Zou\#, Guifang Zeng, Chaonong Cai, Peiping Li, Jiafan Chen, Decheng Li, \\ Baimeng Zhang, Jian Li
}

Department of Hepatobiliary Surgery and Liver Transplantation Center, The Fifth Affiliated Hospital of Sun Yat-sen University, Zhuhai, China Contributions: (I) Conception and design: E Lin, B Zou, J Li, C Cai, B Zhang; (II) Administrative support: J Li, C Cai; (III) Provision of study materials or patients: E Lin, B Zou, G Zeng; (IV) Collection and assembly of data: E Lin, B Zou, G Zeng, P Li, J Chen; (V) Data analysis and interpretation: E Lin, B Zou, G Zeng, D Li; (VI) Manuscript writing: All authors; (VII) Final approval of manuscript: All authors.

\#These authors contributed equally to this work as joint first authors.

Correspondence to: Jian Li, MD. Department of Hepatobiliary Surgery and Liver Transplantation Center, The Fifth Affiliated Hospital of Sun Yat-sen University, 52 Mei Hua East Road, Zhuhai 519000, China. Email: lijian5@mail.sysu.edu.cn.

\begin{abstract}
Background: The pathogenesis of non-cirrhotic hepatocellular carcinoma (HCC) with a high recurrence remains controversial, while microvascular invasion (MVI) is highly suggestive of tumor recurrence. This study aimed to investigate the effects of liver fibrosis on MVI and prognosis in HCC.

Methods: Based on the data of HCC in the Surveillance, Epidemiology, and End Results (SEER) database [2004-2015], multivariate logistic regression was used for correlation analysis. Survival was analyzed by LogRank test and Cox regression, and decision curve analysis and receiver operating characteristic curves were established to evaluate alternative diagnostic and prognostic strategies.

Results: The study included 1,492 patients with MVI (17.8\%) or without MVI (82.2\%) for HCC with a solitary nodule. Liver fibrosis was significantly correlated with the occurrence of MVI, and the risk of MVI in patients with a fibrosis score F5-6 was lower than in those with a score of F0-4 (OR =0.651, 95\% CI: 0.492-0.860). Combining liver fibrosis could improve the prediction performance of MVI risk models, but liver fibrosis was less associated with survival outcomes in comparison with other tumor characteristics.

Conclusions: Lower liver fibrosis correlated with a higher risk of MVI in HCC with a solitary nodule and was a good indicator for improving the performance of MVI risk models. However, it was not a prognostic sensitive indicator.
\end{abstract}

Keywords: Hepatocellular carcinoma (HCC); liver fibrosis; microvascular invasion (MVI); prognosis; Surveillance, Epidemiology, and End Results (SEER)

Submitted Jun 04, 2021. Accepted for publication Aug 06, 2021.

doi: $10.21037 / \mathrm{atm}-21-3731$

View this article at: https://dx.doi.org/10.21037/atm-21-3731

\section{Introduction}

Hepatocellular carcinoma (HCC) is one of the most common malignant tumors globally, accounting for about $90 \%$ of adult primary liver cancer. It is the fourth leading cause of cancer death and seventh leading cause of cancer morbidity worldwide. In 2018 there were 841,000 new liver cancer patients and 781,000 deaths, accounting for $4.7 \%$ and $8.2 \%$ of all cancer incidences and deaths respectively $(1,2)$. In the past three decades, the incidence of HCC has been rising, and may continue to do so until 2030 (3). It 
is worth noting that although the incidence of $\mathrm{HCC}$ in western countries is only $7.5 \%, 15-20 \%$ of HCC in this cohort develop within a non-cirrhotic liver background (NCL) (4).

HCC develops in a NCL less commonly that in one which is cirrhotic. In the absence of a cirrhosis background, the risk of liver failure after surgical resection is reduced, and the 5 -year survival after surgery is about $50 \%$. However, the disease-free survival of HCC in NCL is about $30 \%$, which means that recurrence is very common (5-11). At present, controversy remains about the relative recurrence trend of HCC in NCL $(12,13)$, possibly because of the perceived low specific oncologic risk or even the lack of awareness of carrying a liver disease, or because the diagnosis of HCC in NCL is made outside any surveillance program and usually at an advanced or symptomatic stage (14). High recurrences are related not only to tumor characteristics at the time of initial diagnosis but also to the condition of the underlying non-tumoral parenchyma which may have subclinical liver disease (5).

Liver fibrosis is a common pathological process seen in various chronic liver diseases which develop into cirrhosis. The key steps are chronic injury, inflammation, fibroblast activation and matrix deposition (15). Excessive deposition of liver extracellular matrix or fibrous connective tissue disrupts liver blood perfusion, leading to liver parenchymal metabolism and internal environment disorders, forming a new inflammatory response and tumorigenic environment $(16,17)$. The comparison of HCC in liver parenchyma between cirrhosis and a completely "healthy" liver is too simplistic and crude, because between these two extremes there are extensive substantial hepatic pathological changes without cirrhosis, including liver fibrosis with $\mathrm{F} 2$ and F3, steatohepatitis with steatosis $>30 \%$, and early hemochromatosis, among others (5). And microvascular invasion (MVI) is an important indicator for evaluating the risk of recurrence (18-20). Hepatocellular carcinoma often is single nodule or multiple nodules at the time of initial diagnosis, and studies have shown that patients with HCC initially diagnosed as multiple nodules have a higher incidence of MVI compared to those with single nodule ( $\mathrm{P}<0.001$, OR $=5.10,95 \%$ CI: 2.47-10.52) (21), and the former also have a worse prognosis for long-term postoperative survival than the latter (22).

Therefore, in order to exclude multiple intrahepatic metastases that may be caused by delayed diagnosis and offset the adverse effects of multiple nodular liver cancer on MVI and prognosis of patients, we conducted a study of pooled data of HCC with solitary nodule from the Surveillance, Epidemiology, and End Results (SEER) database maintained by the National Cancer Institute, to explore whether the degree of liver fibrosis affects the risk of MVI and prognosis in HCC. We present the following article in accordance with the STROBE reporting checklist (available at https://dx.doi.org/10.21037/atm-21-3731).

\section{Methods}

\section{Data sources and research cobort}

This study used retrospective data collected from the US National Cancer Research SEER database for analysis. The database was co-built by 18 registries in various states of the United States and contains information on the incidence and outcomes of tumor patients accounting for approximately $28 \%$ of the US population. Available data includes demographic data, including age, gender, race, and the patient's birthplace, as well as tumor information (histology and grade), and general surgery, radiotherapy and other treatment data. Certain data such as AJCC stage, details of surgical treatment, tumor size, and lymph node involvement, could only be queried recently. The SEER database provides many samples for analyzing tumor characteristics, based on wide population coverage and high data accuracy.

Using data collected by the SEER database between 2004 and 2015, HCC patients with solitary nodules were identified. Inclusion criteria: (I) patients diagnosed with HCC (ICD-0-3: 8170-8175) by the World Health Organization (WHO) Classification of Tumors of the Digestive System (edition 2010), and HCC was the primary tumor. (II) Age $\geq 18$ years. (III) A solitary nodule was present. (IV) Surgical resection or liver transplantation was performed, and histopathology was confirmed postoperatively. Exclusion criteria: (I) tumors with mixed types or uncertain origins in histopathology. (II) Incomplete basic information (age, race, gender, diagnosis time, etc.). (III) Incomplete clinical and pathological indicators (including tumor size, tumor differentiation, liver fibrosis score, serum alpha-fetoprotein, vascular invasion, lymph node, and extrahepatic metastasis). (IV) Incomplete followup information (survival time and survival outcome). The detailed selection exclusion criteria and the number of patients are shown in Figure 1. The study was conducted in accordance with the Declaration of Helsinki (as revised in 2013). 


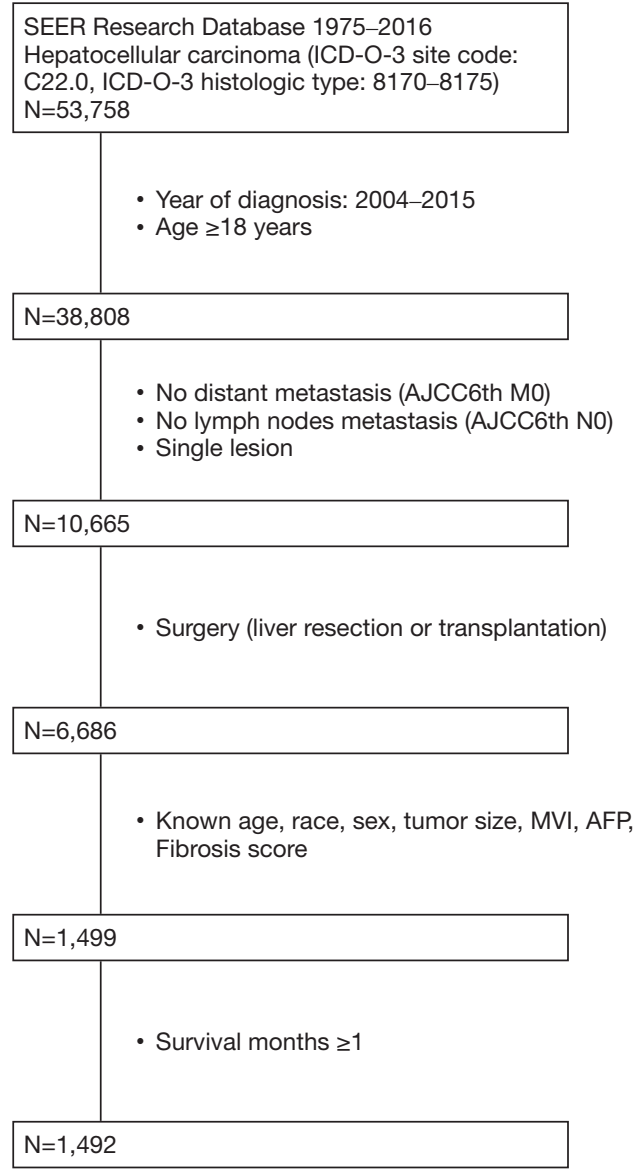

Figure 1 Flow chart for selection of the study population. SEER, Surveillance, Epidemiology, and End Results; ICD-O-3, International Classification of Diseases for Oncology, 3rd edition; AJCC, American Joint Committee on Cancer; MVI, microvascular invasion; AFP, alpha fetal protein.

\section{Clinical variables}

Clinicopathological parameters such as age, sex, race, tumor size, alpha fetoprotein (AFP), fibrosis score, and microvascular invasion (MVI) are included in the SEER database. The fibrosis stage was divided into F0-4 (none to moderate fibrosis) and F5-6 (severe fibrosis or cirrhosis) according to the Ishak score, as indicated by SEER.

\section{Follow-up}

Postoperative survival time, survival status, and cause of death are included in the SEER database. Overall survival (OS) was defined as death from any cause or end of followup after surgery, and cancer specific survival (CSS) was defined as postoperative death due to HCC or end of follow-up. The deadline for follow up was November 2018.

\section{Statistical analysis}

Clinical data were collected through SEER*stat (version 8.3.6) software, SPSS 21.0, and R version3.6.0 ( $\mathrm{R}$ development core team 2011) software was used for statistical analysis. Pearson $\chi^{2}$ test was used for univariate analysis to obtain the related predictors of MVI in HCC patients with a solitary nodule and logistic regression was used for multivariate analysis to obtain the independent predictors of MVI. Kaplan-Meier method was used to draw survival curves, and Log-Rank test was subsequently used to compare the survival curves of each group. A Cox proportional hazards model was applied to the analysis of risk factors that could potentially affect the prognosis, and decision curve analysis (DCA) and receiver operating characteristic (ROC) curves served as suitable methods for evaluating alternative diagnostic and prognostic strategies. The R package 'rmda' was used for the DCA which could evaluate the influence of liver fibrosis and other clinicopathological characteristics on the incidence of MVI and the prognosis of HCC. ROC curves were constructed, and the AUC (area under the curve) was calculated to make the comparison between MVI risk models intuitively, using the package of 'pROC'. $\mathrm{P}<0.05$ was considered to indicate statistical significance (all $\mathrm{P}$ value were derived from twotailed test).

\section{Results}

\section{General characteristics}

After screening, 1,492 HCC patients with solitary nodules were obtained. Among them, 265 patients (17.8\%) with MVI were detected, while 1,227 patients $(82.2 \%)$ did not have MVI. Among all the included patients, 553 patients (37.1\%) had a fibrosis score of $\mathrm{F} 0-4$, and 939 patients (62.6\%) had a fibrosis score of F5-6. The median followup period was 42 months, and the average follow-up period was 52.18 months (from 1-155 months).

\section{MVI related factors}

To explore the predictors of MVI in HCC, we compared the clinical variables of patients. The results showed that MVI was more common among patients with tumor size 
Table 1 Univariate analysis of MVI in HCC patients with a solitary nodule

\begin{tabular}{|c|c|c|c|c|c|}
\hline Characteristics & Subgroups & Proportion of MVI (+) & OR & $95 \% \mathrm{Cl}$ & $P$ value \\
\hline Age (years) & $\geq 60$ & $19.5 \%(160 / 821)$ & & & \\
\hline \multirow[t]{2}{*}{ Race } & White & $15.8 \%(144 / 909)$ & 1.227 & $1.061-1.419$ & $0.006^{\star}$ \\
\hline & Black & $16.9 \%(27 / 160)$ & & & \\
\hline \multirow[t]{2}{*}{ Sex } & Female & $16.8 \%(64 / 380)$ & 1.089 & $0.800-1.484$ & 0.587 \\
\hline & Male & $18.1 \%(201 / 1112)$ & & & \\
\hline \multirow[t]{2}{*}{ Tumor size $(\mathrm{cm})$} & $\leq 2$ & $10.8 \%(34 / 315)$ & 2.018 & $1.375-2.962$ & $<0.001^{*}$ \\
\hline & $>2$ & $19.6 \%(231 / 1177)$ & & & \\
\hline \multirow[t]{2}{*}{ Fibrosis score } & $\mathrm{FO}-4^{\ddagger}$ & $22.6 \%(125 / 553)$ & 0.6 & $0.459-0.785$ & $<0.001^{*}$ \\
\hline & $\mathrm{F} 5-6^{\S}$ & $14.9 \%(140 / 939)$ & & & \\
\hline
\end{tabular}

${ }^{*}$ Chi-square test was used, $\mathrm{P}$ value of $<0.05$ was considered as significant. ${ }^{\dagger}$ American Indian/AK Native, Asian/Pacific Islander. ${ }^{\ddagger}$ Fibrosis score 0-4 (none to moderate fibrosis). ${ }^{\S}$ Fibrosis score 5-6 (severe fibrosis or cirrhosis). AFP, alpha fetoprotein; MVI, microvascular invasion; MVI (+), presence of MVI; OR, odds ratio; $95 \% \mathrm{Cl}, 95 \%$ confidence interval.

Table 2 Multivariate analysis of MVI in HCC patients with a solitary nodule

\begin{tabular}{lcccc}
\hline Characteristics & Subgroups & OR & $95 \% \mathrm{Cl}$ & P value \\
\hline RACE & White vs. others $^{\dagger}$ & 0.742 & $0.550-1.001$ & 0.05 \\
& Black vs. others $^{\dagger}$ & 0.664 & $0.410-1.076$ & 0.096 \\
Tumor size $(\mathrm{cm})$ & $>2$ & 1.790 & $1.210-2.648$ & $0.004^{*}$ \\
AFP & Positive & 1.956 & $1.459-2.622$ & $<0.001^{*}$ \\
Fibrosis score & F5-6 $^{\ddagger}$ & 0.651 & $0.492-0.860$ & $0.003^{*}$ \\
\hline
\end{tabular}

${ }^{*}$ Binary logistic regression model was used, $\mathrm{P}$ value of $<0.05$ was considered as significant. ${ }^{\dagger}$ American Indian/AK Native, Asian/Pacific Islander. ${ }^{\ddagger}$ Fibrosis score 5-6 (severe fibrosis or cirrhosis). AFP, alpha fetoprotein; OR, odds ratio; 95\% Cl, 95\% confidence interval.

$>2 \mathrm{~cm}$, AFP positive, and a fibrosis score of $\mathrm{F} 0-4(\mathrm{P}<0.05)$. The incidence in white and black Americans were similar, both of which were significantly lower than that seen in other races (American Indian/AK Native, Asian/Pacific Islander) (Table 1).

Through multivariate regression analysis, tumor size, AFP, and fibrosis scores were seen to still significantly affect the occurrence of MVI in HCC patients with solitary nodules (Table 2). Further, a tumor size $>2 \mathrm{~cm}$ was 1.790 times more likely to be associated with MVI than if $\leq 2 \mathrm{~cm}$, and patients who were AFP positive were 1.956 times more likely than negative patients. It was worth noting that less MVI occurred in patients with a fibrosis score of F5-6 compared to F0-4, which was statistically significant with OR value of 0.651 (95\% CI: 0.492-0.860). This indicated a higher risk of MVI in HCC patients with slighter liver fibrosis.

DCA findings showed liver fibrosis, tumor size, and AFP had a similar effect on the risk of MVI (Figure 2). ROC curves were constructed and the AUC was calculated, and among the tested MVI risk models, combined with and without liver fibrosis displayed an AUC of 0.63 and 0.60 , respectively $(\mathrm{P}<0.05)$ (Figure 3). These results indicated that the prediction performance of MVI risk models could be 


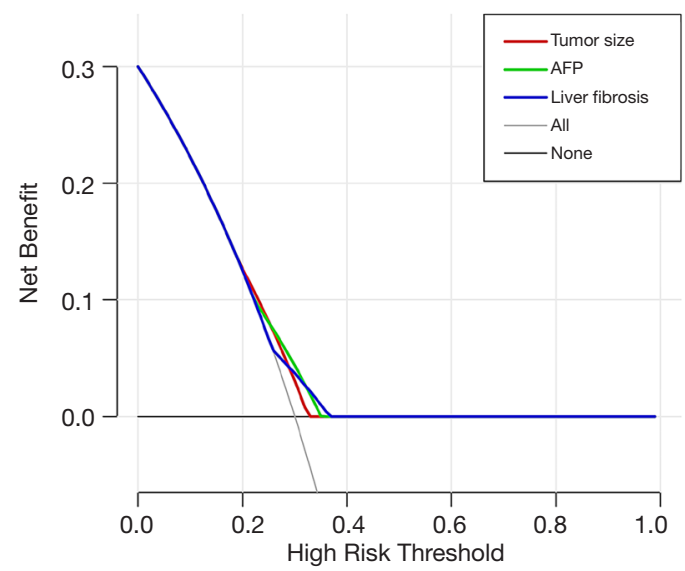

Figure 2 Decision curve analysis in HCC patients with a solitary nodule. The net benefit plotted using fibrosis, tumor size, AFP for MVI. HCC, hepatocellular carcinoma; MVI, microvascular invasion; AFP, alpha fetal protein.

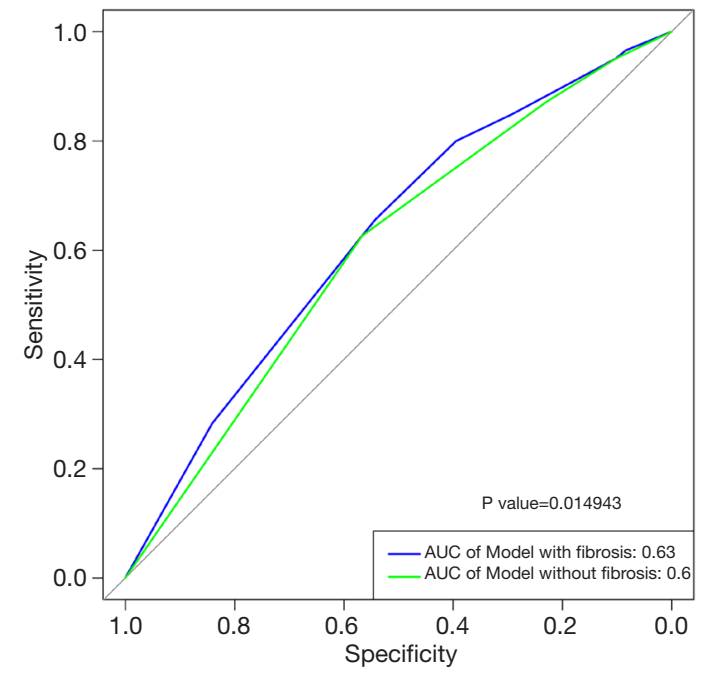

Figure 3 Comparative ROC analysis of MVI risk models combined with or without fibrosis. ROC, receiver operating characteristic; MVI, microvascular invasion; AUC, area under curve.

improved by combining with liver fibrosis.

\section{Survival related factors}

In patients with MVI, the 1-, 3-, 5-year OS were $86.7 \%$, $59.8 \%$, and $51.9 \%$, respectively and the corresponding rate for CSS were $89.9 \%, 71.0 \%$, and $66.2 \%$. The 1-, 3-, 5 -year OS for patients without MVI were $93.7 \%, 79.3 \%$, and $70.2 \%$, respectively and the corresponding rate for CSS were $96.6 \%, 88.0 \%$, and $81.6 \%$. Kaplan-Meier showed that OS and CSS were statistically different between patients with and without MVI (all $\mathrm{P}<0.05$ ) (Figure 4).

The OS of patients with a fibrosis score of F5-6 was $92.4 \%, 76.1 \%$, and $68.0 \%$ at 1,3 , and 5 years, respectively while the corresponding rate for CSS was $95.8 \%, 85.4 \%$, and $80.0 \%$. In patients with a fibrosis score of $\mathrm{F} 0-4$, the 1-, 3-, 5-year OS were 92.5\%, 75.4\%, and 65.1\%, respectively and the corresponding rate for CSS were $94.8 \%, 84.5 \%$, and $77.2 \%$. Kaplan-Meier showed that there was no statistically significant difference in OS between patients with a fibrosis score of F5-6 and F0-4 (P>0.05), but the CSS between the two was statistically different $(\mathrm{P}<0.05)$ (Figure 5).

Cox proportional hazards model analyzes indicated that age ( $\geq 60$ years old), race (black, white $v s$. other), tumor size (>2 cm), AFP (positive), and MVI (positive) were the independent risk factors affecting OS in HCC patients with a solitary nodule (all $\mathrm{P}<0.05)$, of which tumor size $(>3 \mathrm{~cm}$ ), AFP (positive), and MVI (positive) were also the independent risk factors affecting CSS in HCC patients with a solitary nodule (all $\mathrm{P}<0.05)$ (Table 3). Further, liver fibrosis was not an independent risk factor for OS nor CSS (all $\mathrm{P}>0.05$ ). The results of DCA showed that liver fibrosis was less associated with survival outcomes in comparison with tumor size, AFP, and MVI (Figure 6), and the ability of OS and CSS prediction was similar between multivariate models combined with or without liver fibrosis (Figure 7).

\section{Discussion}

MVI is an important reference basis for evaluating the risk of HCC recurrence and selecting post-operative therapy (11,23-26). This study first observed the relationship between liver fibrosis and MVI in HCC patients with a solitary nodule from 2004 to 2015, which was based on the advantages of the SEER database's multi-center and large sample. We tried to reduce the error caused by the delayed diagnosis by controlling the number of tumors, and the results showed that the degree of liver fibrosis was associated with the occurrence of MVI $(\mathrm{P}<0.05$, $\mathrm{OR}=0.651$ ), and the proportion of MVI patients with a fibrosis score of F0-4 and F5-6 were $22.6 \%$ and $14.9 \%$, respectively. It was obvious that the incidence of MVI with no to moderate liver fibrosis was higher than severe fibrosis or cirrhosis in HCC patients with a solitary nodule, which might be one of the reasons for the high recurrence rate of 


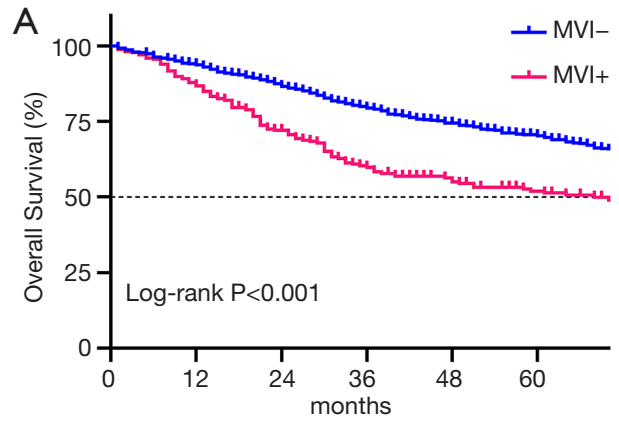

Number at risk

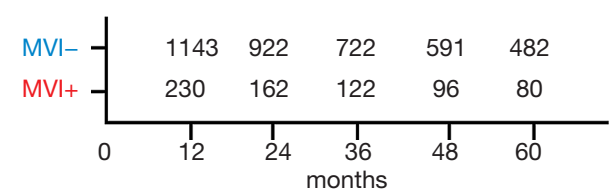

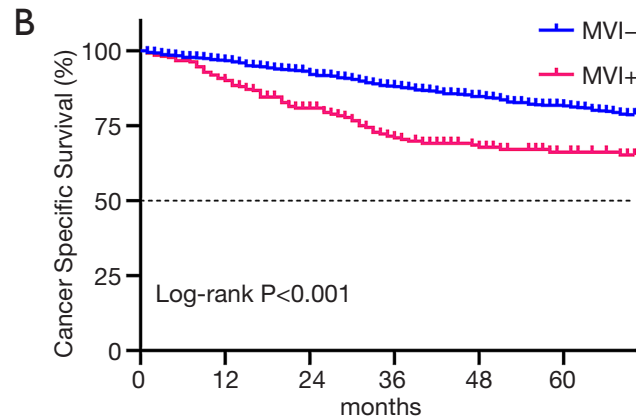

Number at risk

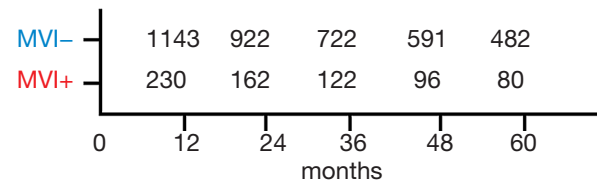

Figure 4 Kaplan-Meier survival curves according to present of microvascular invasion. Impact of MVI on (A) OS and (B) CSS in HCC patients with a solitary nodule. MVI+, presence of MVI; MVI-, absence of MVI. MVI, microvascular invasion; OS, overall survival; CSS, cancer specific survival; HCC, hepatocellular carcinoma.
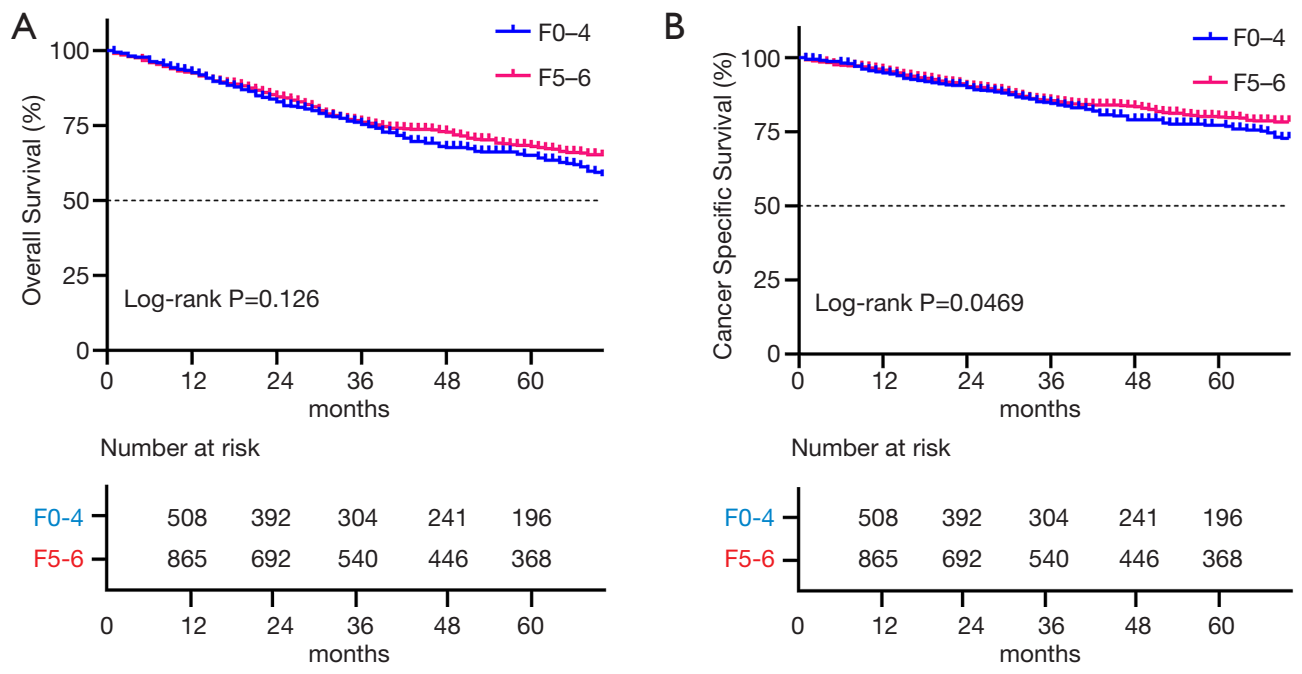

Figure 5 Kaplan-Meier survival curves according to degree of liver fibrosis. Impact of fibrosis score on (A) OS and (B) CSS in HCC patients with a solitary nodule. F0-4, fibrosis scores 0-4 (none to moderate fibrosis); F5-6, fibrosis scores 5-6 (severe fibrosis or cirrhosis). OS, overall survival; CSS, cancer specific survival; HCC, hepatocellular carcinoma.

\section{HCC in NCL.}

Therefore, after the delayed diagnosis caused by irresistible factors was excluded, the effect of different microenvironments on tumor progression caused by liver fibrosis was worthy of our further discussion. A "seed and soil" hypothesis exists with regard to the mechanism of tumor metastasis. Many previous studies have focused on determining how cancer cells (seed) as metastases while ignoring the role of the tumor microenvironment (soil).

When hepatocytes are affected by inflammation or other damage, hepatic stellate cells (HSC) are activated and can be transformed into proliferative myofibroblast-like cells. The cells proliferate in large numbers and secrete extracellular matrix. The damaged area is encapsulated by matrix and fibrous scar tissue, intrahepatic fibrosis is formed, and liver structure is reconstructed; activated HSC can also increase 


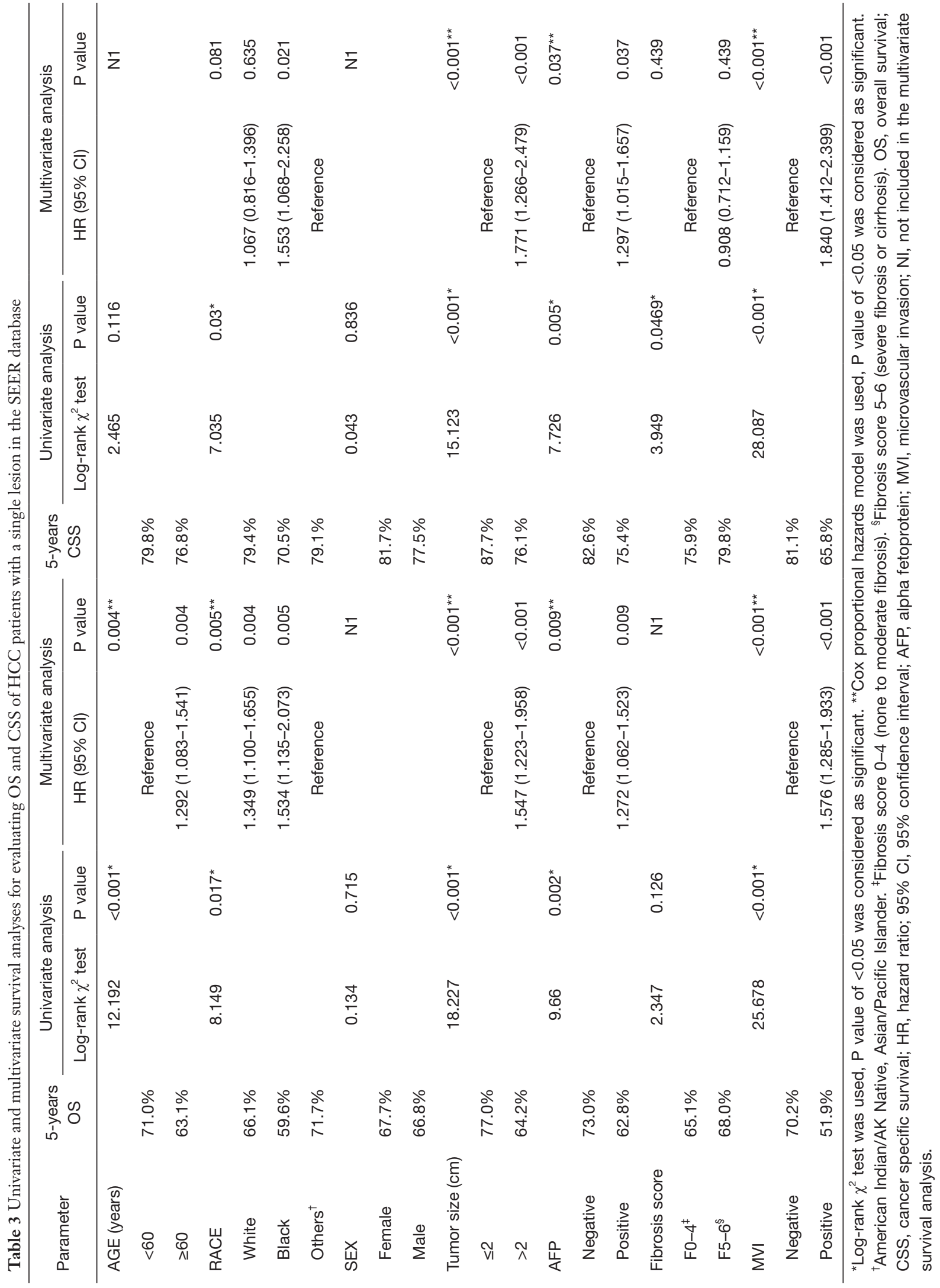



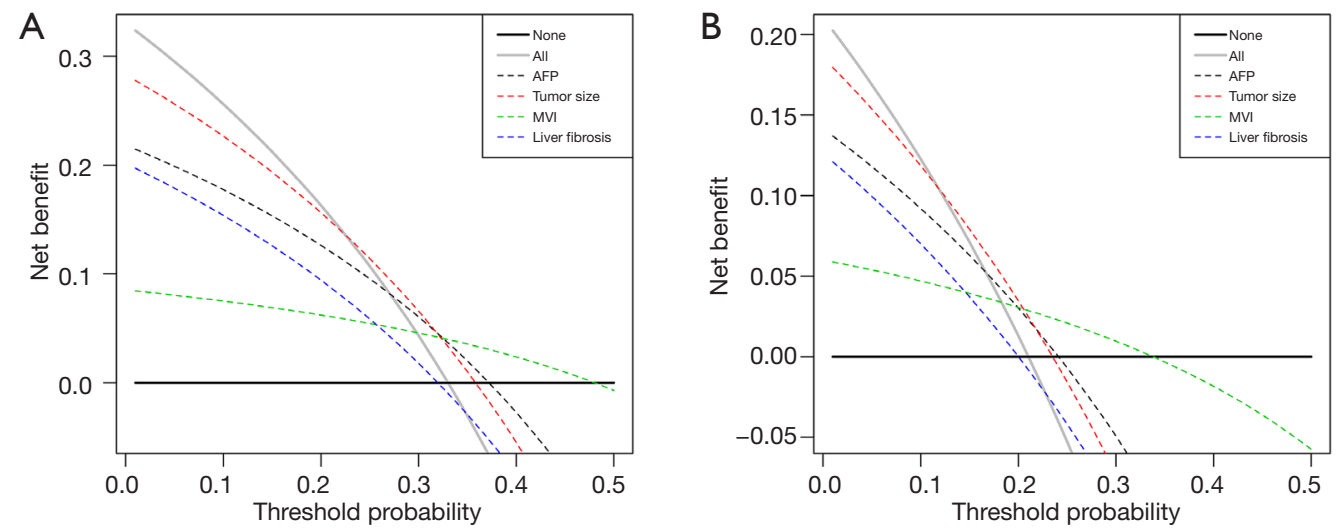

Figure 6 Decision curve analysis in HCC patients with a solitary nodule. (A) The net benefit plotted using fibrosis, tumor size, and AFP for overall survival. (B) The net benefit plotted using fibrosis, tumor size, and AFP for cancer specific survival. HCC, hepatocellular carcinoma; MVI, microvascular invasion; AFP, alpha fetal protein.
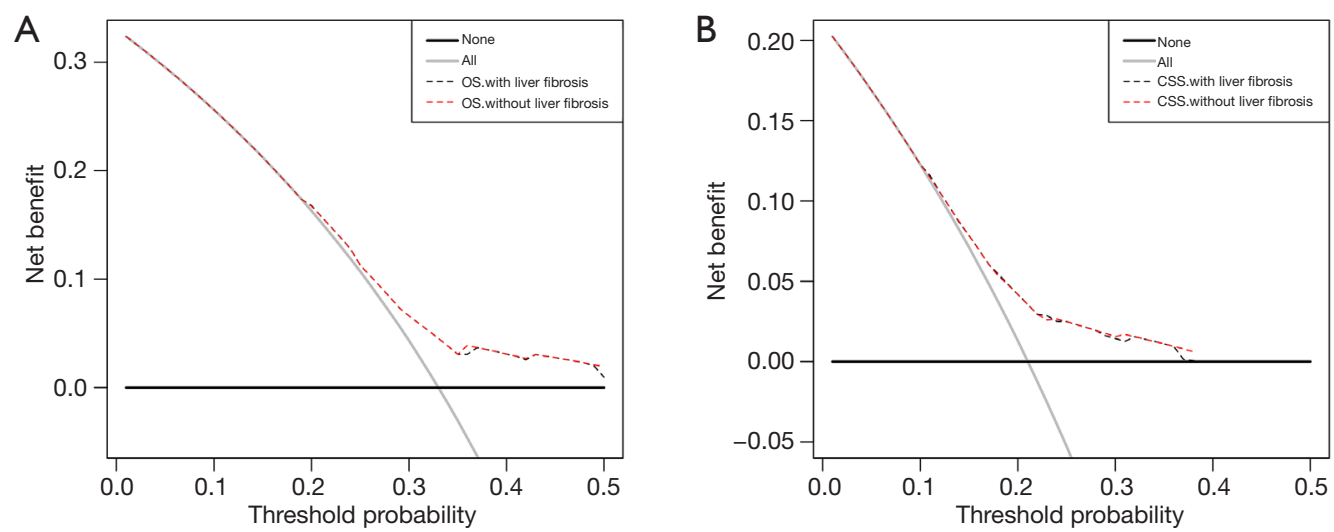

Figure 7 Decision curve analysis in HCC patients with a solitary nodule. (A) The net benefit plotted using multivariate models with and without fibrosis for overall survival. (B) The net benefit plotted using multivariate models with and without fibrosis for cancer specific survival. HCC, hepatocellular carcinoma; OS, overall survival; CSS, cancer specific survival.

intrahepatic sinusoidal pressure through cell contraction, disrupting intrahepatic hemodynamics; in addition, due to the presence of tissue matrix metalloproteinases inhibitors, the excessive extracellular matrix accumulated under the basement membrane and inner membrane cannot be digested completely, and the endothelial cell window pores are significantly reduced, which leads to obstacles in the exchange of substances between blood and hepatocytes $(27,28)$.

The activation of hepatic stellate cells (HSC) is an important step in the development of HCC with cirrhosis. On the contrary, the pathogenic role of HSC is suppressed in HCC with non-cirrhosis. The reduced activation of HSC and delayed formation of the fibrosis interval may result in tumors and neovascularization not being restricted by extensive fibrous scars (29-31), which may cause a bigger tumor size, which is significantly positively correlated with the risk of MVI $(32,33)$. Extravascular histological differences may also affect the permeability of new blood vessels, and related reports have shown that liver cirrhosis can reduce the risk of liver metastasis (34-36).

There were some studies showed that the lower degree of fibrosis in the adjacent liver tissues of HCC was related to the larger tumor diameter (the tumor diameters of fibrosis F0-2 and F3-4 were about $10.1 \pm 6.7$ and $6.6 \pm 3.5 \mathrm{~cm}$, respectively). $26 \%$ (8/31) of HCC with mild liver fibrosis lacked a complete capsule, but all the HCC with a high degree of fibrosis had a complete capsule (12). In addition, compared with HCC with cirrhosis, HCC with NCL had 
more obvious satellite nodules and vascular invasion, but the difference was not statistically significant (12).

Although the risk of MVI was significantly increased in lower degree of liver fibrosis of HCC patients, we could see no significant difference in the survival prognosis between patients with different degree of liver fibrosis through the comparison of survival analysis. This may be due to the 'congenital advantage' of non-cirrhotic hepatocellular carcinoma (with better liver reserve function), and the reresection of intrahepatic recurrences had been proven to be equally safe and effective (5). Therefore, for patients with HCC in non-cirrhosis liver, the key to improve the survival outcome is to offset the negative impact of the high incidence of MVI through accurate preoperative prediction and close postoperative follow-up. On the one hand, various preoperative methods could be used to predict the risk of MVI and guide clinicians to choose more active treatment options during the operation, such as wide resection margin, R0 resection, anatomical resection, etc. On the other hand, for HCC patients with non-cirrhosis, attention should be paid to active monitoring after diagnosis and active treatment after recurrence. Although recurrence was frequent in the first two years after surgery, as it may occur within 4 to 5 years after surgery, surveillance should continue for more than 5 years (5).

The DCA and ROC curve comparison showed that tumor size, AFP, and liver fibrosis had a similar effect on the risk of MVI, and the addition of liver fibrosis could improve the performance of MVI risk models. Until now there have been few preoperative MVI prediction models that include the degree of fibrosis (37-40). Although the degree of liver fibrosis involved in our study was diagnosed by postoperative pathology, many methods are available to determine this before surgery with high accuracy, such as ultrasound and magnetic resonance elastography (41-43). The use of these and other measures may improve the accuracy of MVI prediction, which can direct clinical treatment.

This study has some limitations, including its retrospective nature. Incomplete information such as preoperative complications, preoperative biochemical indicators, and surgical protocols might have all affected the detection rate of vascular invasion and in-depth discussion on the etiology. Further, as the results are based on a United States population, their broader application is uncertain and needs to be determined according to multi-center and large sample research in individual countries. However, the conclusions drawn by this study should raise awareness on behalf of clinicians of HCC in NCL. In these patients, it is necessary to develop an individual treatment that differs from cirrhotic HCC and pay more attention to customizing surveillance to improve prognosis and survival. In noncirrhotic HCC patients with high-risk factors for MVI (large tumor diameter, metabolic diseases such as hypertension, diabetes, hyperlipidemia, and family history of nonalcoholic fatty liver disease) $(44,45)$, whether a wider resection margin or corresponding post-operative adjuvant therapy could obtain a better survival prognosis requires further investigation.

\section{Conclusions}

In summary, the results of this study showed that liver fibrosis was an independent predictor of MVI in HCC patients with a solitary nodule, and lower liver fibrosis correlated with a higher risk of MVI. Furthermore, liver fibrosis was a good indicator that could improve the performance of MVI risk models but was less associated with survival outcomes in comparison with other strong prognostic factors.

\section{Acknowledgments}

We are grateful for the help of the support team from SEER at the National Cancer Institute.

Funding: This work was supported by the National Natural Science Foundation of China (grant number 81971773); the Natural Science Foundation of Guangdong Province (grant number 2018A0303130292); the Guangdong Basic and Applied Basic Research Foundation (grant number 2019A1515011356, 2020A1515010203); the Science and Technology Planning Project of Guangdong Province of China (grant number 2019A030317023); the Medical Scientific Research Foundation of Guangdong Province of China (grant number A2019099); the Fundamental Research Funds for the Central Universities, Sun Yat-sen University (grant number 20ykpy51, 2021qntd35); the Science and Technology Planning Project of Zhuhai city of China (grant number 20191211E030097); and the COVID-19 Infection Prevention and Control Emergency Technology Project of Zhuhai city (grant number ZH22036302200020PWC).

\section{Footnote}

Reporting Checklist: The authors have completed the STROBE reporting checklist. Available at https://dx.doi. org/10.21037/atm-21-3731 
Conflicts of Interest: All authors have completed the ICMJE uniform disclosure form (available at https://dx.doi. org/10.21037/atm-21-3731). The authors have no conflicts of interest to declare.

Etbical Statement: The authors are accountable for all aspects of the work in ensuring that questions related to the accuracy or integrity of any part of the work are appropriately investigated and resolved. The study was conducted in accordance with the Declaration of Helsinki (as revised in 2013). The authors signed the Data-Use Agreement for the 1975-2017 SEER Research Data File. This study was deemed exempt by the Ethics Committee and Institutional Review Board of The Fifth Affiliated Hospital of Sun Yat-Sen University, as it is based on the data extracted from the publicly available SEER database.

Open Access Statement: This is an Open Access article distributed in accordance with the Creative Commons Attribution-NonCommercial-NoDerivs 4.0 International License (CC BY-NC-ND 4.0), which permits the noncommercial replication and distribution of the article with the strict proviso that no changes or edits are made and the original work is properly cited (including links to both the formal publication through the relevant DOI and the license). See: https://creativecommons.org/licenses/by-nc-nd/4.0/.

\section{References}

1. Bray F, Ferlay J, Soerjomataram I, et al. Global cancer statistics 2018: GLOBOCAN estimates of incidence and mortality worldwide for 36 cancers in 185 countries. CA Cancer J Clin 2018;68:394-424.

2. Tang A, Hallouch O, Chernyak V, et al. Epidemiology of hepatocellular carcinoma: target population for surveillance and diagnosis. Abdom Radiol (NY) 2018;43:13-25.

3. Petrick JL, Kelly SP, Altekruse SF, et al. Future of Hepatocellular Carcinoma Incidence in the United States Forecast Through 2030. J Clin Oncol 2016;34:1787-94.

4. Zhang $\mathrm{Y}$, Wang $\mathrm{C}, \mathrm{Xu} \mathrm{H}$, et al. Hepatocellular carcinoma in the noncirrhotic liver: a literature review. Eur J Gastroenterol Hepatol 2019;31:743-8.

5. Alkofer B, Lepennec V, Chiche L. Hepatocellular cancer in the non-cirrhotic liver. J Visc Surg 2011;148:3-11.

6. Bège T, Le Treut YP, Hardwigsen J, et al. Prognostic factors after resection for hepatocellular carcinoma in nonfibrotic or moderately fibrotic liver. A 116-case European series. J Gastrointest Surg 2007;11:619-25.
7. Lang H, Sotiropoulos GC, Brokalaki EI, et al. Survival and recurrence rates after resection for hepatocellular carcinoma in noncirrhotic livers. J Am Coll Surg 2007;205:27-36.

8. Lee DH, Lee JM. Primary malignant tumours in the noncirrhotic liver. Eur J Radiol 2017;95:349-61.

9. Barinaga M. Designing therapies that target tumor blood vessels. Science 1997;275:482-4.

10. Yang T, Lin C, Zhai J, et al. Surgical resection for advanced hepatocellular carcinoma according to Barcelona Clinic Liver Cancer (BCLC) staging. J Cancer Res Clin Oncol 2012;138:1121-9.

11. Iguchi T, Shirabe K, Aishima S, et al. New Pathologic Stratification of Microvascular Invasion in Hepatocellular Carcinoma: Predicting Prognosis After Living-donor Liver Transplantation. Transplantation 2015;99:1236-42.

12. Paradis V, Zalinski S, Chelbi E, et al. Hepatocellular carcinomas in patients with metabolic syndrome often develop without significant liver fibrosis: a pathological analysis. Hepatology 2009;49:851-9.

13. Leung C, Yeoh SW, Patrick D, et al. Characteristics of hepatocellular carcinoma in cirrhotic and non-cirrhotic non-alcoholic fatty liver disease. World J Gastroenterol 2015;21:1189-96.

14. Giannini EG, Marenco S, Bruzzone L, et al. Hepatocellular carcinoma in patients without cirrhosis in Italy. Dig Liver Dis 2013;45:164-9.

15. Tacke F, Weiskirchen R. An update on the recent advances in antifibrotic therapy. Expert Rev Gastroenterol Hepatol 2018;12:1143-52.

16. Lee YA, Wallace MC, Friedman SL. Pathobiology of liver fibrosis: a translational success story. Gut 2015;64:830-41.

17. Weiskirchen R, Tacke F. Liver Fibrosis: From Pathogenesis to Novel Therapies. Dig Dis 2016;34:410-22.

18. Rodríguez-Perálvarez M, Luong TV, Andreana L, et al. A systematic review of microvascular invasion in hepatocellular carcinoma: diagnostic and prognostic variability. Ann Surg Oncol 2013;20:325-39.

19. Feng LH, Dong H, Lau WY, et al. Novel microvascular invasion-based prognostic nomograms to predict survival outcomes in patients after $\mathrm{R} 0$ resection for hepatocellular carcinoma. J Cancer Res Clin Oncol 2017;143:293-303.

20. Lim KC, Chow PK, Allen JC, et al. Microvascular invasion is a better predictor of tumor recurrence and overall survival following surgical resection for hepatocellular carcinoma compared to the Milan criteria. Ann Surg 2011;254:108-13.

21. Lei Z, Li J, Wu D, et al. Nomogram for Preoperative 
Estimation of Microvascular Invasion Risk in Hepatitis B Virus-Related Hepatocellular Carcinoma Within the Milan Criteria. JAMA Surg 2016;151:356-63.

22. Midorikawa Y, Takayama T, Higaki T, et al. Comparison of the surgical outcomes in patients with synchronous versus metachronous multiple hepatocellular carcinoma. Biosci Trends 2021;14:415-21.

23. Eguchi S, Takatsuki M, Hidaka M, et al. Predictor for histological microvascular invasion of hepatocellular carcinoma: a lesson from 229 consecutive cases of curative liver resection. World J Surg 2010;34:1034-8.

24. Fujita N, Aishima S, Iguchi T, et al. Histologic classification of microscopic portal venous invasion to predict prognosis in hepatocellular carcinoma. Hum Pathol 2011;42:1531-8.

25. Rodríguez-Perálvarez M, Luong TV, Andreana L, et al. A systematic review of microvascular invasion in hepatocellular carcinoma: diagnostic and prognostic variability. Ann Surg Oncol 2013;20:325-39.

26. Shirabe K, Toshima T, Kimura K, et al. New scoring system for prediction of microvascular invasion in patients with hepatocellular carcinoma. Liver Int 2014;34:937-41.

27. Pellicoro A, Ramachandran P, Iredale JP, et al. Liver fibrosis and repair: immune regulation of wound healing in a solid organ. Nat Rev Immunol 2014;14:181-94.

28. Yang N, Shi JJ, Wu FP, et al. Caffeic acid phenethyl ester up-regulates antioxidant levels in hepatic stellate cell line T6 via an Nrf2-mediated mitogen activated protein kinases pathway. World J Gastroenterol 2017;23:1203-14.

29. Baffy G, Brunt EM, Caldwell SH. Hepatocellular carcinoma in non-alcoholic fatty liver disease: an emerging menace. J Hepatol 2012;56:1384-91.

30. Friedman SL. Mechanisms of hepatic fibrogenesis. Gastroenterology 2008;134:1655-69.

31. Hytiroglou P, Park YN, Krinsky G, et al. Hepatic precancerous lesions and small hepatocellular carcinoma. Gastroenterol Clin North Am 2007;36:867-87, vii.

32. Ünal E, İdilman İS, Akata D, et al. Microvascular invasion in hepatocellular carcinoma. Diagn Interv Radiol 2016;22:125-32.

33. Zhang X, Li J, Shen F, et al. Significance of presence of microvascular invasion in specimens obtained after surgical treatment of hepatocellular carcinoma. J Gastroenterol Hepatol 2018;33:347-54.

34. Dahl E, Rumessen J, Gluud LL. Systematic review with meta-analyses of studies on the association between cirrhosis and liver metastases. Hepatol Res 2011;41:618-25.

35. Gervaz P, Pak-art R, Nivatvongs S, et al. Colorectal adenocarcinoma in cirrhotic patients. J Am Coll Surg 2003;196:874-9.

36. Lv Y, Zhang HJ. Effect of Non-alcoholic Fatty Liver Disease on the Risk of Synchronous Liver Metastasis: Analysis of 451 Consecutive Patients of Newly Diagnosed Colorectal Cancer. Front Oncol 2020;10:251.

37. Wang L, Jin YX, Ji YZ, et al. Development and validation of a prediction model for microvascular invasion in hepatocellular carcinoma. World J Gastroenterol 2020;26:1647-59.

38. Yang L, Gu D, Wei J, et al. A Radiomics Nomogram for Preoperative Prediction of Microvascular Invasion in Hepatocellular Carcinoma. Liver Cancer 2019;8:373-86.

39. Deng G, Yao L, Zeng F, et al. Nomogram For Preoperative Prediction Of Microvascular Invasion Risk In Hepatocellular Carcinoma. Cancer Manag Res 2019;11:9037-45.

40. Gao SX, Liao R, Wang HQ, et al. A Nomogram Predicting Microvascular Invasion Risk in BCLC 0/ A Hepatocellular Carcinoma after Curative Resection. Biomed Res Int 2019;2019:9264137.

41. Petitclerc L, Sebastiani G, Gilbert G, et al. Liver fibrosis: Review of current imaging and MRI quantification techniques. J Magn Reson Imaging 2017;45:1276-95.

42. Lurie Y, Webb M, Cytter-Kuint R, et al. Non-invasive diagnosis of liver fibrosis and cirrhosis. World J Gastroenterol 2015;21:11567-83.

43. Zhang YN, Fowler KJ, Ozturk A, et al. Liver fibrosis imaging: A clinical review of ultrasound and magnetic resonance elastography. J Magn Reson Imaging 2020;51:25-42.

44. Liu Y, Li H, Ye N, et al. Non-Cirrhotic Liver is Associated with Poor Prognosis of Hepatocellular Carcinoma: A Literature Review. Med Sci Monit 2019;25:6615-23.

45. Ertle J, Dechêne A, Sowa JP, et al. Non-alcoholic fatty liver disease progresses to hepatocellular carcinoma in the absence of apparent cirrhosis. Int J Cancer 2011;128:2436-43.

(English Language Editor: B. Draper)

Cite this article as: Lin E, Zou B, Zeng G, Cai C, Li P, Chen J, Li D, Zhang B, Li J. The impact of liver fibrosis on microvascular invasion and prognosis of hepatocellular carcinoma with a solitary nodule: a Surveillance, Epidemiology, and End Results (SEER) database analysis. Ann Transl Med 2021;9(16):1310. doi: 10.21037/atm-21-3731 